\title{
MR Brain Image Segmentation Optimized by Using Ant Colony Algorithm with BrainSeg3D for Multiple Sclerosis Tumors Detection
}

\author{
Dalenda Bouzidi \\ National Engineering \\ School of Sfax (ENIS) \\ University of Sfax, \\ Tunisia \\ Laboratory of Technology \\ for Smart System (LT2S) \\ Digital Research Center of \\ Sfax (CRNS), Tunisia \\ dadou dalenda@,hotmail.fr
}

\author{
Fahmi Ghozzi \\ National School of Electronics \\ and Telecommunications of \\ Sfax, University of Sfax, \\ Tunisia \\ Laboratory of Technology \\ for Smart System (LT2S) \\ Digital Research Center of \\ Sfax (CRNS), Tunisia \\ fahmi.ghozzi@enetcom.usf.tn
}

Khaled Taouil

National School of Electronics and Telecommunications of

Sfax, University of Sfax, Tunisia

Laboratory of Technology

for Smart System (LT2S),

Digital Research Center of

Sfax (CRNS), Tunisia

khaled.taouil@enetcom.usf.tn

\author{
Ahmed Fakhfakh \\ National School of Electronics \\ and Telecommunications of \\ Sfax, University of Sfax, \\ Tunisia \\ Laboratory of Technology \\ for Smart System (LT2S), \\ Digital Research Center of \\ Sfax (CRNS), Tunisia \\ ahmed.fakhfakh@enetcom.usf.tn
}

Received: November 10, 2020. Revised: December 8, 2020. Accepted: December 10, 2020. Published: December 14, 2020.

\begin{abstract}
Nowadays, Brain tumors segmentation is a very important task in diagnosis of brain lesions. There are diversapproaches of segmentation, but segmentation of brain tumors is always difficult due to the complex features of magnetic resonance imaging MRI such as the appearance of vague and unclear tumors and boundaries. In this paper, an automatic MRI segmentation method is used to solve these problems. Here, tumor segmentation is treated as a problem of classification using the Ant Colony ACO optimization algorithm combined with a proposed protocol based on BrainSeg3D tools. Many studies and many existing approaches tend the multiple sclerosis (MS) which is a chronic inflammatory anomaly of the central nervous system. To pick up the outliers of multiple sclerosis, we should do a diagnosis by magnetic resonance imaging with various sequences. For this aim, some late technics of segmentation and classification of MRI images have been suggested for the automatic detection of MS outliers. In this work, we submit a novel protocol based on current and novel semi-automated tumors segmentation technics of BrainSeg3D. Evaluation of our results was performed on novel MR database containing 30 MS patients, which were learned with a 3T MR scanner with conventional sequences so that to estimate our assessment with the acquired consensus segmentation with ground truth data. The submitted algorithm is evaluated by using MATLAB GUI program and BrainSeg3D tools.
\end{abstract}

Keywords-Multiple sclerosis, Lesions, segmentation, ACO, BrainSeg3D, noveldataset, Consensus

\section{INTRODUCTION}

$\mathrm{M}$ ULTIPLE sclerosis (MS) is a chronic inflammatory-

demyelinating pathology of the central nervous system. MRI detects outliers in MS patients with elevated sensitivity but low specificity, and is used for diagnosis, prognostication and as a substitute marker in MS essays [1]-[2]. The symptoms encountered are then very diverse.

They can be driving, decreasing the strength of one or more members, altering the coordination or voluntary control of certain movements, or generating fatigue. It may have visual, respiratory, urinary, psychological symptoms associated with varying degrees. It is traditional to say that the evolution of MS is unpredictable. It is true if we consider a particular patient, but if we study several thousand sick, it becomes clear that MS can be classified into some forms clinical trials. The evolution of which has specific characteristics, allowing group patients with these characters in common. The form with pure pushes, called "remittance" is characterized by the appearance abruptness of one or more symptoms that gradually disappear in six to eight weeks, leaving no trace. These outbreaks can occur with varying frequency, depending on each patient, ranging from several outbreaks per year to episodes spaced by several years apart.

The form with push and progression, called "remittentprogressive," evolves of remittent, without sequelae, for 10 to 15 years on average and the patient keep a certain handicapoutbreaks continue even though they are often spaced.

The "secondary progressive" form concerns patients having first of allpresented a relapsing form and in whom the flares disappear completely aftera certain amount of time to make room for a slow, more or less regular progressiondisability.

The progressive form from the start, called "primary progressive" as opposedto the previous form, begins insidiously, most often bywalking problems. These disorders worsen slowly and other discreetly over time. At no time do these patients therefore present a marked aggravation or improvement of their condition. The following figure illustrates the different known forms of clinical evolution of multiple sclerosis.

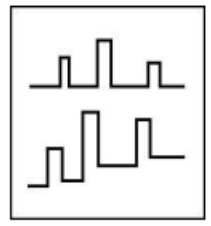

Forme RR

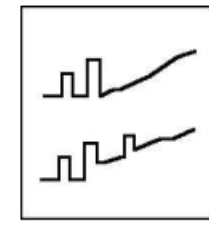

Forme SP

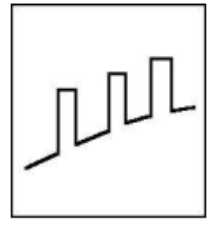

Forme PR

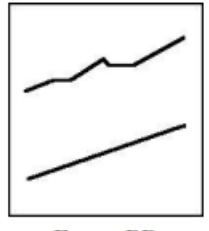

Forme PP
Fig.1. Diagram of the different known forms of clinical evolution of multiple sclerosis. RR: recurrent-remitting form; SP: secondaryprogressive form; PR: progressive-relapsing form; PP: primaryprogressive form 
The diagnosis of multiple sclerosis (MS) is based on a body of clinical and para-clinical evidence of temporal and spatialdissemination of central nervous system (CNS) lesions apart from another progressive disease. Thus the identification of MS can be completed before the existence of two outbreaksor a single outbreak with spatio-temporal dissemination criteria established by MRI.

Conventional MRI in MS typically includes T2-weighting (T2-w), proton density (PD), fluid-attenuated inversion recovery (FLAIR), and T1-weighting (T1-w) with and without enhancement of gadolinium [1]-[3]. MS outliers can occur in any substance in the central nervous system, but on conventional MRI, gray matter (GM) MS tumors have a signal intensity equivalent to the intensity of GM emerge normal surrounding and therefore, other specialized sequences are required to discover GM lesions [1]-[4]. In contrast, white matter (WM) tumors are classified as hyper-intense compared to the normal neighboring emerging WM on the T2-w, FLAIR and PD sequences [5].

The primary object of segmenting medical images is to division it into divers anatomical organizations, thus isolation the components of interest, such as brain outliers, and divers tissues. Segmentation of medical images nowadays is a harder problem thanks to bass contrast, poor resolution, and presence of artifact noise, involved borders, and patient motion.MRI has a significant action on the identification and knowing of multiple sclerosis. MRI can also be used to test the efficacy of a processing and to make retrospective investigations enabling a improved accordance of the pathology. But that does not preclude having other solutions permitting a better robustness, reliability and performance to spot the tumors of the disease. In addition, the re-productivity of manual segmentation approaches is frequently very mediocre. Many study teams are actively working there and literature is ample. However, no method yields total satisfaction today.

In this study, the segmentation protocol is applied to the MRI brain with a novel dataset that include $30 \mathrm{MS}$ patients. The goal of this article is to test and assimilate impartially our proposed segmentation protocol using ACO algorithm [6]-[15] combined with a protocol based on the Brain Seg3D software tools [7]. Our motivation is to detect MS lesions for different patients included in novel MRI datasets.

The residual of this study is orderly as follows: we briefly present in section II some previous works; in section III we will detail the public database we used for the test and the validation of our algorithm. Then section IV presents the proposed algorithm based on ACO combined with a proposed protocol by using the tools of BrainSeg3D software. Section V presents some results and discussion. Finally, we would end up with a conclusion and submit some perspectives.

\section{PREVIOUS SEGMENTATION TECHNIQUES}

MS outliers can be segmented manually; however, this work can be fastidious and time consuming. Even more critical to the process of detecting tumors and to the contour of lesions which leads to great intra and inter-evaluator variability, an infamous characteristic of manual segmentation of outliers[8]-[9]-[10]. Therefore, the routine of manual segmentations for a long time, are not sufficiently exact and established for the separation of biomarkers [11]. Automated approaches have been intensively industrialized in the past two decenniums to provide more literal and coherent segmentation of outliers [12]-[13]. The main characteristics of automated approaches reside in their robustness to MR acquisition shortcoming (MR bias range, partial volume impact and image sound), and the great biological variability of cerebral anatomy and pathology of tumors.

Despite an important methodological advancement which prohibits raising the major challenges, it is not yet determined which approach or even a class of automated approaches (for example unsupervised and supervised) can be considered as a standard for the extract of biomarkers [14]. A standard approach to segmentation of lesions can only be confirmed on the basis of objective and rigorous validation of standard data sets with very specific segmentation of lesions. Despite known shortcomings, most researchers still use manual contouring of lesions [13].

There are different other segmentation technics disposable in the literature. The best known approaches of classification are the mobile center algorithm or K-Means [16], fuzzy partitioning or FCM [17] and genetic algorithms [18]. C. Senthil Singh et al. [19] proposed a computer-assisted system for cerebral MRI segmentation to pick up tumor localization using a K-means classification algorithm attended by morphological filtration. This method takes it possible to segment the outliers from various cerebral MR images and to avert sorely collected regions. But it generates different marks for a different number of groups. We can also refer to the work of carass et al [20]which lay out the thresholding approaches were applied together with the voxel connectivity analysis and proposed Lesion-TOADS which is an atlas-based segmentation method for the brain and tumors by using a topological and statistical atlases conjoint with fuzzy clustering, while in other research propound in [21] used an automatic threshold for the FLAIR image to tap outliers correspondent to keen areas. However, in their tumor segmentation tool (LST), Schmidt et al. [22] employed a threshold on a probabilistic lesion map calculated on the basis of cerebral segmentation and refined by a region growth algorithm.

Other researchers have propound a new automatic method of segmentation could be applied first for cerebral zones differentiation, and then to the characterization and localization of outliers in MS. On the other hand, this method proposed the combination of MR Images based on the wavelet method. This combination would require one single image resume all the required report related to the current tumors present in both white and gray matters. In addition, the proposed method assume into account the textural, spatial and 
statistical characteristics, which were extracted using the Grayscale Path Length Matrix (GLRL) and the Volumetric Grayscale Co-occurrence Matrix (GLCM), in addition to certain shape characteristics (asymmetry and kurtosis). From the series of all the extracted characteristics, the submitted approach tempted to conserve the optimal ones, including the most discriminating ones, thus presenting a relevant segmentation. This optimization was performed by using the conjoint of SVM tool with a genetic algorithm (GA) [23].

Another unsupervised technique implicated an opening brain segmentation providing by an atlas used GMM.

The most recent segmentation study presents a new protocol to develop benchmark segmentations of white matter tumors based on multi-rater consensus using a new MR database of 30 patients with multiple sclerosis. On these databases, three specialist reviewers individually segmented white matter lesions; using semi-automated tumor contouring tools developed in-house [24]. They have developed specialized BrainSeg3D software which allows their correct and effective delineation in 3D MR images. The BrainSeg3D is clearly available, downloadable from http://lit.fe.uni-lj.si/tools.

\section{Public Database}

Among the current public datasets, we can find a public MRI simulator named BrainWeb [25] which contains three synthetical images T1w, T2w and PDw, the BrainWeb dataset is employed only to supply evidence of theory for novel segmentation approaches.

The initial openly obtainable database of clinical MR images was established for the object of a defiance on MS outliers segmentation [10]. This database contains 52 cases of patients with MS tumors with a standard brain MR T1w, T2w and FLAIR sequences on two Siemens $3 \mathrm{~T}$ scanners on various sites.

Another database of clinical MR images of patients with multiple sclerosis includes 20 cases of MS patients, each imagined at 3 to 5 time points by a 3T MRI scanner using the classic T1w, T2w, PDw and FLAIR sequences. In total, there were 80 data sets and in each, tumors were manually segmented by two trained evaluators. This dataset was distributed as part of a challenge on the longitudinal segmentation of lesions [26].

The current challenge on segmentation of MS outliers [27] adduces 53 databases from 4 dive sites and $43 \mathrm{~T} / 1.5 \mathrm{~T}$ MRI scanners for divers. Each kit contains FLAIR, pre and postcontrast T1w and DP / T2w sequences. In each case, seven liberated specialists segmented the outliers manually and consensual segmentation was created by merging the segmentations based on automatic LOP STAPLE method [28]. The major recent challenge consists on white-matter outliers segmentation [24] applied on MR databases of 30 patients with multiple sclerosis, which were learned on a 3T MR scanner with conventional sequences. A cohort of $30 \mathrm{MS}$ patients was envisaged by a $3 \mathrm{~T}$ Siemens Magnetom Trio MR system at the University of Medical Center Ljubljana
(UMCL). Each one of patient's MR scans consisted of a 2D T1-weighted, T2-weighted and a 3D FLAIR image. On these databases three specialist evaluators executed segmentations of WMLs, and then they modified varied mixed sessions to make consensus-based gold standard segmentation.

TableI. IRM IMAGE OF DATASETSETTING

\begin{tabular}{llll}
\hline & T1 & T2 & FlalR \\
\hline Dimension & 20 & 20 & 30 \\
\hline Repetitiontime(TR) & $2000 \mathrm{~ms}$ & $6000 \mathrm{~ms}$ & $5000 \mathrm{~ms}$ \\
\hline Echotime(TE) & $20 \mathrm{~ms}$ & $120 \mathrm{~ms}$ & $392 \mathrm{~ms}$ \\
\hline Inversiontime(TI) & $800 \mathrm{~ms}$ & & $1800 \mathrm{~ms}$ \\
Flipangle(FA) & $120^{\circ}$ & $120^{\circ}$ & $120^{\circ}$ \\
Sampling & $0.42 \times 0.42 \times 3.30 \mathrm{~mm}$ & $0.57 \times 0.57 \times 3.00-$ & $0.47 \times 0.47 \times 0.80 \mathrm{~mm}$ \\
& & $3,30 \mathrm{~mm}$ & \\
\hline
\end{tabular}

The idea was to allow reviewers to critically reassess their segmentations and come to an agreement on a specialist judgment about what is and what is not a lesion.

Prior showing our propound algorithm assent to automatic segmentation to detect tumors in cerebral MR images, we describe the novel database employed.

The assessment of the submitted segmentation method was carried out using one existent dataset,incorporating divers MS phases: relapsing-remitting, clinical isolated syndrome, secondary progressive and primary progressive.

In this study, we based on the new MR database of 30 patients with MS lesions founded by Lesjak et.al [24]-[30], obtained by a $3 \mathrm{~T}$ Siemens Magnetom Trio MR system at the University of Medical Center Ljubljana (UMCL). Each one of patient's MR scans incorporated of 2D T1-w, 2D T2- w and a 3D FLAIR image (Table I).

Every one of these images has been segmented into three classes: the gray matter class $(G)$, the white matter class (WM) and the cerebrospinal fluid class (CSF), . The next figure shows the $30 \mathrm{MS}$ patients of the dataset images. 

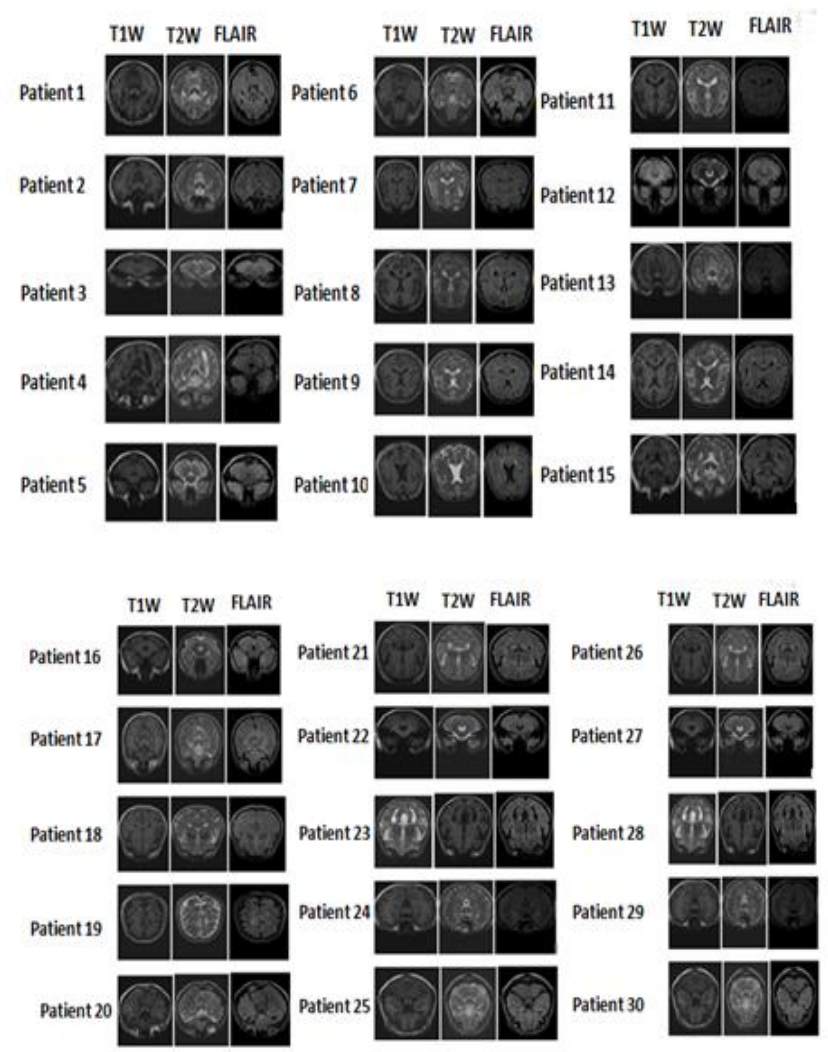

Fig.2. MRI image of the 30 patients of database

Differentiating these tumors related to MS based only on the MR images is hard and patient's clinical aspects and background (e.g. cerebro-vascular risk factors) may assist determine the tumor origin. Since the major part of patients were immature (median age was 39 years, cf. Table II), with clinically-definite diagnosis of MS and without clinical confirmation of other sickness related to white-matter tumors, the experts did not explicitly consider the tumor origin.

\section{TableII. PATIENT DEMOGRAPHIC AND TREATMENT} INFORMATION

\begin{tabular}{llll}
\hline Gender & Age & MS phenotype & Therapy \\
\hline 23 female & 25 to 64 years & $24 \mathrm{RR}$ & 1 Avonex \\
7 male & Median:39 years & $2 \mathrm{SP}$ & 3 Betaferon \\
& (female 39, male 33) & $1 \mathrm{PR}$ & 1 Copaxone \\
& $2 \mathrm{CIS}$ & 1 Extavia \\
& 1 unspecified & 5 Gilenya \\
& & 1 Myfenax \\
& & 2 Rebif \\
& & 6 Tysabri \\
& & 1 unspecified \\
& & 9no therapy \\
\hline
\end{tabular}

RR-relapsing remitting; SP-secondary progressive; PR-progressive relapsing; CIS-clinically isolated syndrome

\section{THE PROPOSED ACO BRAIN SEG3D ALGORITHM}

\section{A. Contributions}

Paper [7] describes an algorithm used the tools of BrainSeg3D software applied on MR datasets of 30 patients with MS tumors, which were learned on a 3T MR scanner with conventional sequences. The idea was to re-evaluate critically segmentations with the consensual segmentation indicates with the novel MR datasets of what isand what is not a lesion.

Also, in the papers [6]-[15]which applied an ACO algorithm on the same database, the authors obtained a good results compared5to ground truth of the novel MR datasets.

This is why we thought to combine these two algorithms to reevaluate our results by assimilating with the reference of this database.

We expose in this paper the suggested method which used the fusion of ACO algorithm with a proposed protocol by using existing and new semi-automated outliers segmentation tools of BrainSeg3D software.

So as to facilitate the tumors segmentation, we employed the specialized BrainSeg3D software which let their specific and effectual delineation in 3D MR images. This software was developed by Lesjak et al [24]. The BrainSeg3D, which is based on an open-source medical image treatment and displaying platform Seg3D [29], supply an interactive local semi-automated segmentation device.

\section{B. ACO with BrainSeg3D tools algorithm}

The proposed algorithm combines two algorithms for processing and segmenting MRI images with multiple sclerosis: the first algorithm is based on Ant Colony Optimization algorithm [6]-[15]. This algorithm was tested by using MATLAB GUI program.

The second proposed protocol uses the processed and optimized images from the first algorithm; the proposed protocol is based on the Brain Seg3D software image processing tools [7]. The flowchart of the proposed algorithm is detailed on figure 3 . 


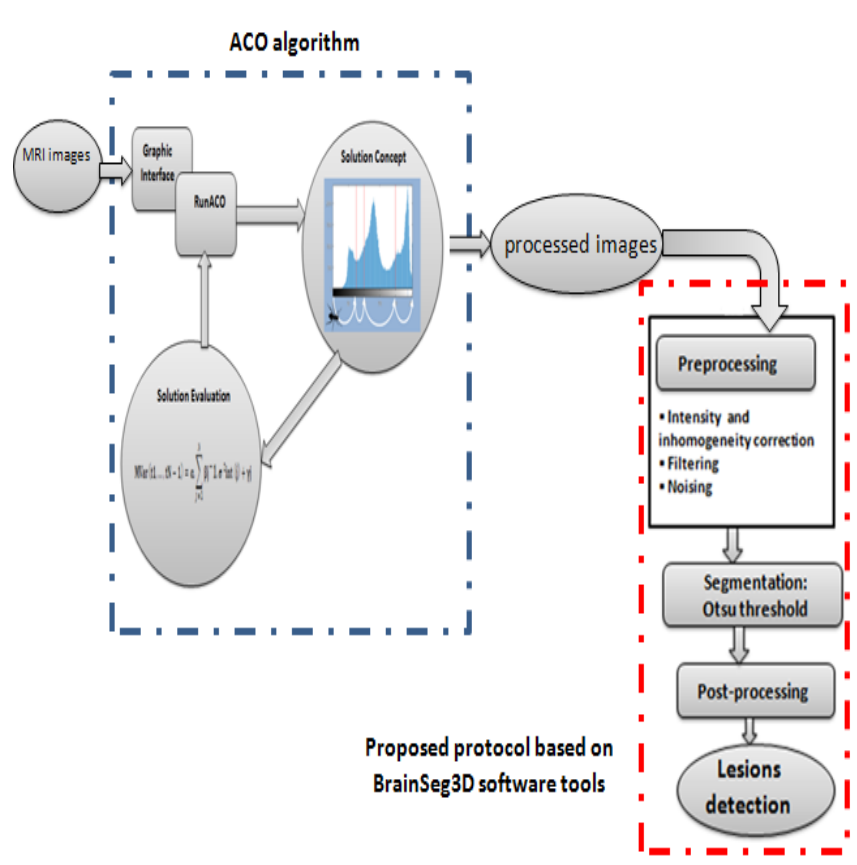

Fig.3. Flowchart of the proposed algorithm

\section{ACO algorithm}

We propose this algorithm in our paper [15] to improve the image segmentation method by maximizing the intraclass variance Mvar. To do this, we use ant colony metaheuristics (ACO). This allows us to get as close as possible to the optimal solution. We use the intraclass variance criterion MVar to which we make modifications [31]. The latter consists of three parameters to better characterize good quality segmentation. Then, the segmentation thresholds maximizing the criterion are sought using the ACO algorithm.

We will explain in following the different steps of the algorithm we used.

The overall functioning of RunACO is based on a set of ants. Each ant will propose segmentation thresholds. The image from these thresholds will be evaluated for each ant, and the best image will be preserved.

The execution is carried out over several rounds, and each round each of the ants will propose a threshold and be assessed.

We can see on the graphic of figure 3 in the part of ACO algorithm an ant building its segmentation thresholds. An image is actually made up of pixels that have values between 0 (black) and 255 (white). It is therefore built on 256 shades of gray. The ant here offers four class of segmentation.

The ant then goes to an evaluation stage, where the relevance of its proposal will be evaluated with a criterion function. The solution will be compared to the other solutions already found, and the program will keep the best one according to the optimization of the best value of the intraclass variance MVar (3). This operation is repeated a certain number of times for each ant. At each iteration, a percentage of the evaporate hormones.
Initially, $\mathrm{k}$ ants will move randomly on gray levels. Then, other ants are allowed to move on the gray levels $n$ times according to a given probability, and deposit a quantity $\Delta \tau \mathrm{i}, \mathrm{j}(\mathrm{t})$ of pheromones at each displacement. $\tau i, j$ represents the probability of jumping from a threshold $i$ to a threshold $j$. So we have the formula:

$$
\Delta \tau i, j(t)=\left\{\begin{array}{lll}
\frac{1}{\mathrm{~L}^{\mathrm{k}}(\mathrm{t})} & \text { if } & (i, j) \in \mathrm{Tk}(\mathrm{t}) \\
0 & \text { if } & (i, j) \notin \mathrm{Tk}(\mathrm{t})
\end{array}\right.
$$

Where $\mathrm{Tk}(\mathrm{t})$ is the solution provided by the ant $\mathrm{k}$ at iteration $t$, and $L^{k}(t)$ is the sum of the segmentation thresholds contained in the solution. This means that on its way, the ant will drop at each of its jumps the inverse of the sum of the thresholds it will have traveled.

At each loop turn, the pheromones evaporate according to the following expression:

$$
\tau \mathrm{i}, \mathrm{j}(\mathrm{t}+1)=(1-\rho) \tau \mathrm{i}, \mathrm{j}(\mathrm{t})+\sum_{k=1}^{m} \Delta \tau \mathrm{i}, \mathrm{j}(\mathrm{t})
$$

where $\Delta \tau i, j(t)$ is the quantity of pheromones deposited by the ant, $m$ the number of ants used at the iteration $t$ and $\rho$ the evaporation rate which is fixed. Initially, pheromones are initialized by a small amount $\tau 0 \geq 0$.

Moreover, at each iteration, the best thresholds will be selected thanks to the MVar criterion:

$$
\operatorname{MVar}(\mathrm{t} 1, \ldots, \mathrm{tN}-1)=\alpha \cdot \sum_{j=1}^{N} \beta \mathrm{j}^{-} 1 . \sigma^{2} \mathrm{int}(\mathrm{j})+\gamma \mathrm{j}(3)
$$

When the stop criterion is exceeded, the algorithm then stops the number of iterations given for which the MVar criterion does not allow to find better segmentation thresholds and does not allow to execute, where $\mathrm{N}$ represents the number classes to segment (assumed to be known a priori), $\sigma^{2}$ int (j) the intraclass variance of the class $\mathrm{j}, \mathrm{t} 1$ to $\mathrm{tN}-1$ the selected segmentation thresholds, and $\beta \mathrm{j}, \gamma \mathrm{j}$ and $\alpha$ the variance correction parameters intraclass used in the Otsumethod [16][33]. $\alpha$ is $((1000 * \mathrm{M})-1 . \sqrt{ } \mathrm{NR})$ where $\mathrm{M}$ is the total number of pixels in the image and NR is the number of regions, that is, the number of related components in the segmented image. This word is worn to satabilize and correct over-segmented images. The term $\beta \mathrm{j}=(1+\log \mathrm{Nj})$ is chosen so that the term $\beta^{-} \mathrm{j} 1 . \sigma^{2}$ int $(\mathrm{j})$ is weak for large classes. $\mathrm{Nj}$ is the number of pixels in class $\mathrm{j}$. The term $\gamma \mathrm{j}=(\mathrm{C}(\mathrm{Nj}) / \mathrm{Nj})$ is high when the segmented image has many regions of the same size, especially if they are small. $\mathrm{C}(\mathrm{Nj})$ is the number of regions whose cardinal is equal to $\mathrm{Nj}$ for large regions, $\mathrm{C}(\mathrm{Nj})$ is, in most cases, equal to 1 , whereas for small regions it becomes greater than 1 .

Concerning this algorithm, our work is mainly focused on the variation of the parameters empirically in order to have good results for the segmentation of the images of the new database and a good detection of MS lesions. 
So, according to the research work of [6], the optimization problem comes from parameter $\beta$ which is very important.

When $\beta$ is so high, we can observe from figure 4 that it prevented us from having relevant images because they were simply unreadable with very small thresholds and we cannot detect any lesion (Fig.4).

(a)
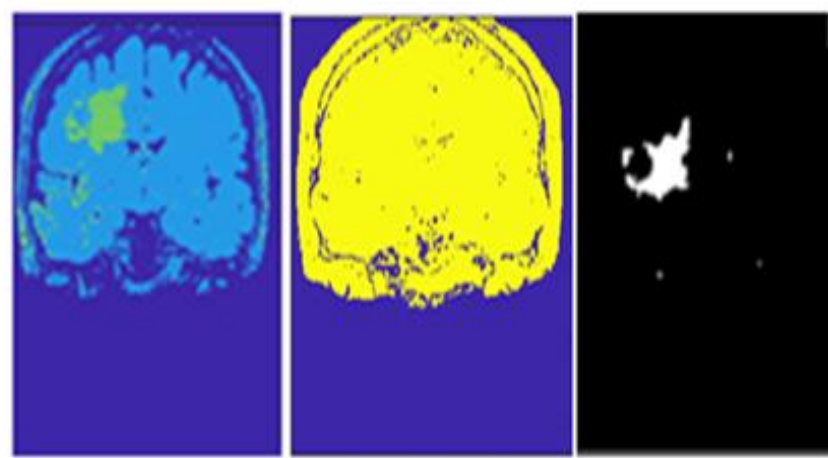

Fig.4. Example of coronal Flair image of patient5: a) segmented image with $\beta=0.5, b)$ segmented image with $\beta=5$, c) ground truth

Next we tested the variation of parameter $\beta$ as a function of DSC similarity coefficient which is detailed in section $\mathrm{V}$ with respect to the ground truth;we obtain the following graph where when we increase the $\beta$ the coefficient of similarity compared to the ground truth will directly decrease so we cannot detect existing MS lesions. In our case, we take $\beta=0.5$ in order to maximize the DSC.

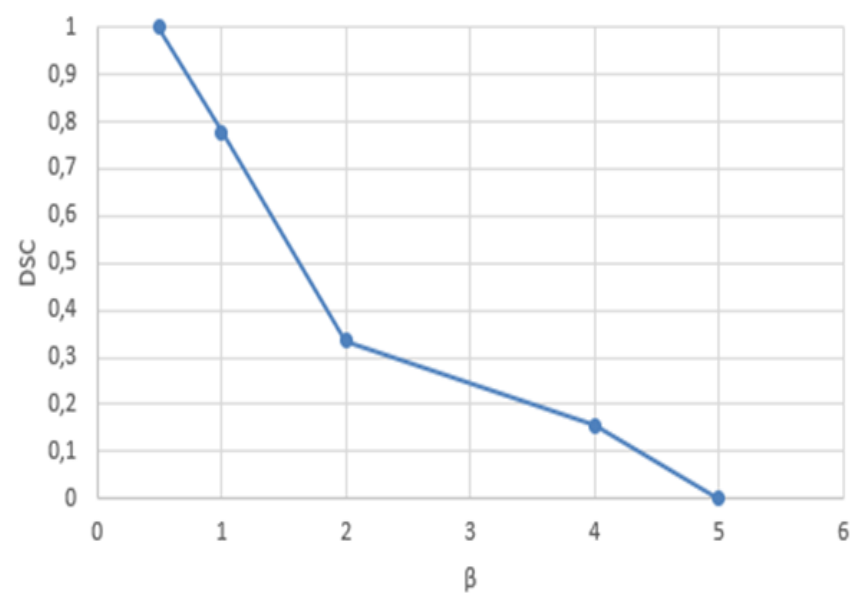

Fig.5. Graph of DSC in terms of $\beta$

Still the number of ants $\mathrm{k}$ is useful for specifying the execution time in order to give the threshold tuples selected by each ant which facilitates the segmentation of the image (Fig.6).

When we increase the number of ants $\mathrm{k}$, the time will also increase easily since the number of iterations will increase for all the ants in order to select the best three segmentation thresholds.

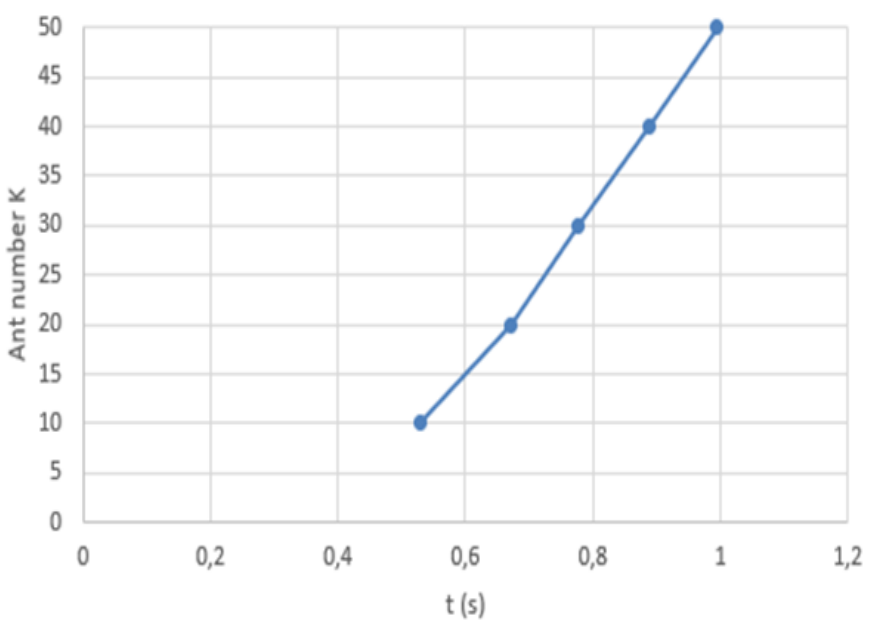

Fig.6. Graph of Ant number $\mathrm{k}$ depending on time $\mathrm{t}$

After the values validation test,for all simulations the next values were selected for the various parameters of the applied method: the number of ants $\mathrm{k}=20$, the evaporation $\operatorname{rate} \rho=0.1$, and the initialization probability $\tau 0=0.01$. The other parameters are shown in Table II.

TableIII. ACO ALGORITHM SETTING

\begin{tabular}{ll}
\hline Setting & Values \\
\hline Ants number k & $20 * \mathrm{~N}$ \\
\hline Threshold number & 3 \\
Evaporation Coefficient $\boldsymbol{\rho}$ & 0.1 \\
$\boldsymbol{\alpha}$ & 1 \\
$\boldsymbol{\beta}$ & 0.5 \\
\hline
\end{tabular}

\section{BrainSeg3D}

The second proposed protocol is applied on the processed and optimized images from the first algorithm. It is divided into three major steps: Preprocessing and post-processing filters as well as automatic segmentation filters acheived in the brainSeg3D software [7].

BrainSeg3D is perform software manufactured for segmentation and displaying of image data. It is employed predominately to load 3D scalar informations, such as MRI or CT. This software yields and sweep mask stickers by recognizing divers regions of interest in the initial image data. Seg3D expedites the operation based on reactive tools such as image dealing filters and manuel masking approaches. This algorithm is applied on the novel database. Previous to segmenting outliers, every patient $\mathrm{T} 1 \mathrm{w}, \mathrm{T} 2 \mathrm{w}$ and FLAIR images are preprocessed. For example, we do the screening of the region of the brain in the T1w image [32], keeped by the mutual information-based registration of the three sequences images. 
Finally, the rectification of inhomogeneity and intensity [33] was effected on every of the covered masks.

We will clarify in the next the usefulness, of every block of our propound protocol.

\section{- Intensity Correction Filter}

The intensity correction filter performs the intensity on the data layer homogeneous.

This filter contains two principal parameters: polynomial order controls the type of spline used to correct the image and edge sensitivity parameter controls the correction algorithm that prevents artifacts from touching the edges of the image.

\section{- Curvature anisotropic diffusion filter}

This filter effects anisotropic diffusion on a data layer used the Modified Curvature Scattering Equation (MCDE). This filter is used to minimize noise in images while protecting special image characteristics, such as edges and can be established on the work of [14]-[34].

This filter contains two essential setting are the iterations parameter will control the number of iterations of this filter. And the Sensitivity Range setting controls the "scatter" of objects in the image.

\section{- Otsuthreshold Filter}

The Otsu threshold filter provides good quick segmentation in many cases as it attempts to group data values in a data layer based on their similarity and create mask layers that identify similar data regions. The only parameter in this implementation is the number of thresholds ( 1 to 4 ), or the number of divisions in the volume.

\section{- Connected Component Filter}

The connected component filter employed to suppress mask data that is separated from a agreed region and thus can minimize noise in segmentation. This filter locates connected data regions in a mask layer that are coincident to the defined seed points.

\section{- Connected ComponentSize Filter}

The connected component size filter enables the employer to operate the attached regions in the volume based on their size. For example, thresholding real data can generate numerous regions of interest that properly mask the data, but can also catch petty regions of noise in other areas of the testing.

\section{- $\quad$ Median Filter}

This is a nonlinear filter that finds the median value of neighboring pixels and uses that value to replace the original pixel. This filter used to reduce noise, as it can also preserve sharp boundaries. For each pixel (except edges), the neighborhood is controlled by the distance parameter. While increasing the distance also increases smoothness.

For all tested results, the subsequent values have been picked and tested empirically for the various parameters of divers filters clarified on top (TABLE IV).

TableIV. VALUES OF DIVERS FILTER PARAMETERS

\begin{tabular}{|l|c|}
\hline \multicolumn{1}{|c|}{ Setting } & Values \\
\hline Polynomial Order & 1 \\
\hline Edge Sensitivity & 0.05 \\
\hline Iterations & 100 \\
\hline Sensitivity Range & 0.5 \\
\hline Threshold number & 4 \\
\hline
\end{tabular}

\section{RESUlTS}

In order to show the performance of our algorithm in terms of Dice Similarity Coefficient DSC, accuracy, robustness of sensitivity, and Total load lesion TLL, our algorithm, in this part, was used for a novel MRI database of 30 patients with multiple sclerosis.

For comparison, we implemented the three algorithms ACO algorithm, propound protocol used the tools of BrainSeg3D software and an algorithm that combines the two techniques together ACO_BrainSeg3D.

The evaluation of our approach used the assimilation of the acquired segmentation and the correspondent reference map for every test image. The next figure (Fig.7) exemplify the results of segmentation used the Otsu threshold tool after preprocessing of patient10 where all outliers probably emerged.

The results of segmentation by the suggestedalgorithmACO_BrainSeg3D are exposed in Fig.8. This algorithm yields well results, where all the lesions have arised in spite of there being falsely spotted lesions.
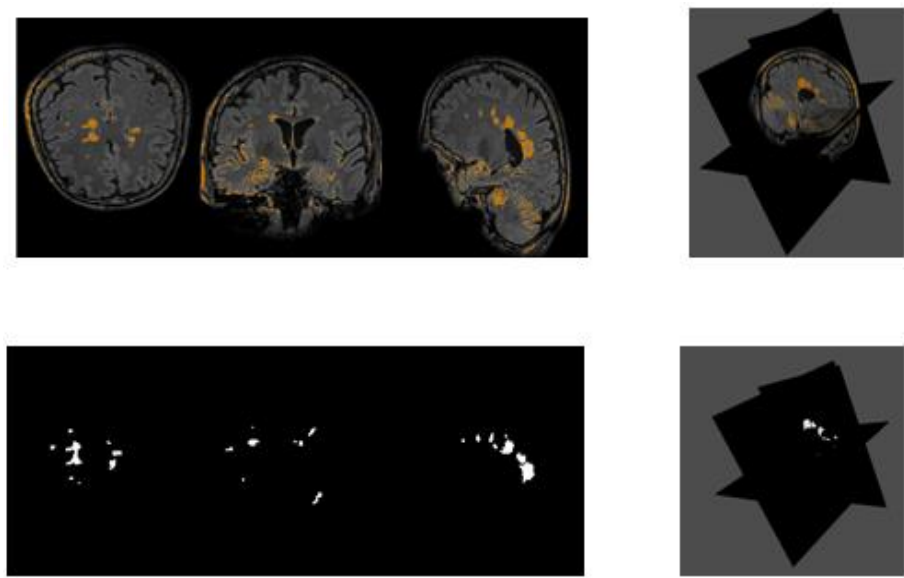

Fig.7.Axial, sagittal and coronal of the FLAIR image, and 3D visualization (from left to right) of consensus segmentation of whitematter lesions for patient10 
Since our essential goal is to spot all outliers, we propound a region spotted as a true positive (TP) if at least one voxel laps our ground truth, other it is enumerated as a false positive (FP). Any ticketing in " ground truth" that is not spotted by our algorithm is posed as a false negative (FN) [35]-[36].

The values of the dice similarity coefficient (DSC) [35] - [36] oscillate in $[0,1]$ and a value greater than 0.6 marks well accordance with the ground of truth:

$\mathrm{DSC}=(2 * \mathrm{TP}) /((2 * \mathrm{TP})+\mathrm{FP}+\mathrm{FN})$

In addition, we calculated the sensitivity defined by:

$$
\text { Sensitivity }=\mathrm{TP} /(\mathrm{TP}+\mathrm{FN})
$$

And the precision that proved the capability to tap every current outliers corresponds to the value:

$$
\text { Precision }=\mathrm{TP} /(\mathrm{TP}+\mathrm{FP})
$$

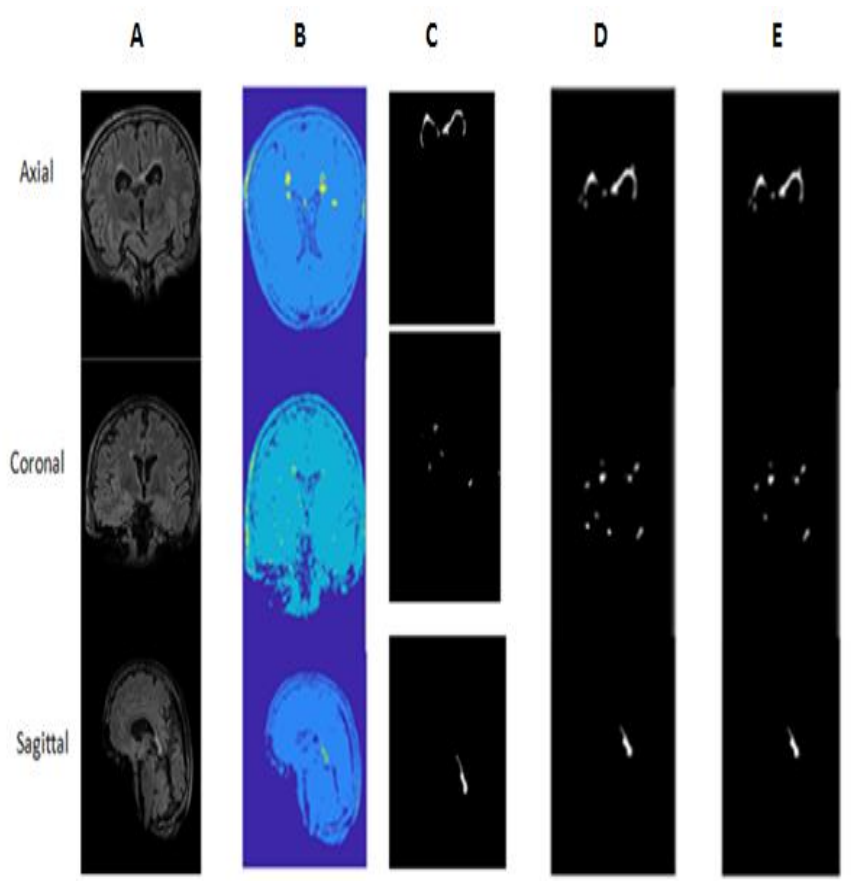

Fig.8.Lesion detection example of patient 10: A) Original pathological image (Axial, coronal and sagittal image cut FLAIR of patient10), B) Our image test of ACO algorithm, C)Our image test of proposed protocol of BrainSeg3D, D)Our image test of proposed algorithm ACO_BrainSeg3D and E) Ground truth

We practice the propound algorithm ACO_BrainSeg3D on the novel database with the $30 \mathrm{MS}$ patients and we computed the coefficient DSC, the sensitivity and the precision as exposed in the TABLE $\mathrm{V}$.

The respective values procured with our proposed algorithm used ACO algorithm with the semi-automated proposed protocol usingBrainSeg3D software tools were 0.799 and 1 . We note that the DSC values acquired in this test are lightly elevated than those reported on every lesion segmentations publicized in the literature (Fig.9).

This is owing to the event that we can see the positions of further lesions in link with the consensus-segmentation, and therefore, we can detect the missed outliers, which could otherwise have a strong impact on DSC.

TableV. VALUESOF DSC,PRECISIONANDSENSITIVITY

\begin{tabular}{llllllllllllllll}
\hline Patient & 1 & 2 & 3 & 4 & 5 & 6 & 7 & 8 & 9 & 10 & 11 & 12 & 13 & 14 & 15 \\
\hline DSC & 0.884 & 0.799 & 0.889 & 0.799 & 0.835 & 0.889 & 0.789 & 0.857 & 0.888 & 0.835 & 0.866 & 1 & 0.896 & 0.888 & 0.858 \\
& & & & & & & & & & & & & & & \\
Precision & 0.95 & 0.72 & 0.86 & 0.68 & 0.8 & 0.9 & 0.7 & 0.81 & 0.89 & 0.8 & 0.86 & 1 & 1 & 0.8 & 0.98 \\
Sensitivity & 0.77 & 0.66 & 0.68 & 0.58 & 0.8 & 0.84 & 0.69 & 0.66 & 0.86 & 0.8 & 0.71 & 1 & 0.7 & 0.74 & 0.87 \\
& & & & & & & & & & & & & & & \\
Patient & 16 & 17 & 18 & 19 & 20 & 21 & 21 & 23 & 24 & 25 & 26 & 27 & 28 & 29 & 30
\end{tabular}

$\begin{array}{llllllllllllllllll}\text { DSC } & 0.860 & 1 & 0.895 & 0.935 & 0.885 & 0.925 & 0.950 & 0.935 & 1 & 0.799 & 0.927 & 0.8 & 0.988 & 0.865 & 1 \\ \text { Precision } & 0.86 & 1 & 1 & 1 & 0.95 & 0.95 & 0.95 & 0.95 & 1 & 0.68 & 0.85 & 1 & 1 & 0.88 & 1\end{array}$

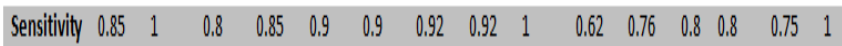

Our values obtained for DSC can be calculated with respect to the consensual DSC segmentation .It is related and admissible in this measurement range compared to precedent work [24] of inter- and intra-rater variability's where the DSC is in the range $0.67-0.73$.

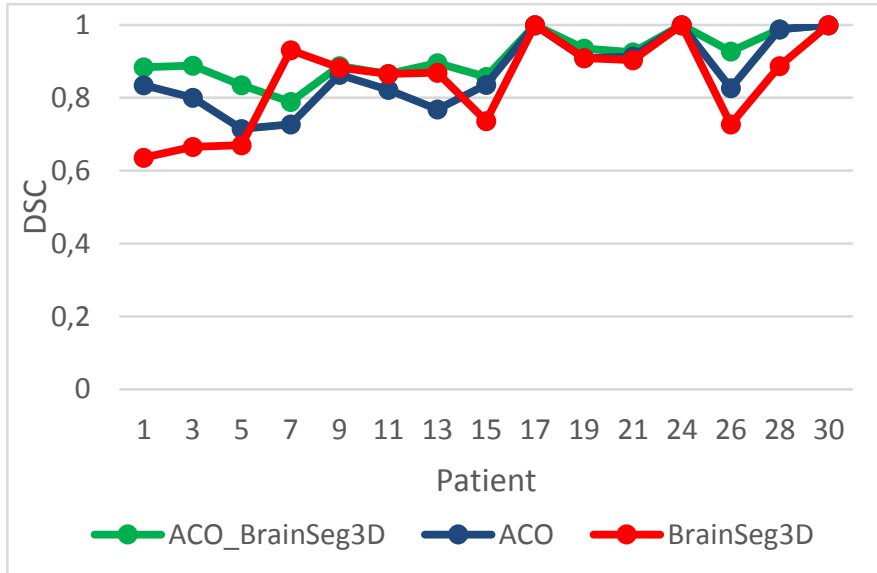

Fig.9. DSC Graph of $30 \mathrm{MS}$ patients of dataset

Again, the values of DSC, precision and sensitivity are very close for the values of two algorithms performed separately, for the first ACO algorithm the values of DSC are between $0.698-1$ and the second protocol used the tools of BrainSeg3D software including the values of DSC are between $0.636-1$ (Fig.10).However, the values of DSC of our proposed algorithm ACO_BrainSeg3D included between 0.799 and 1 .

The average DSC value for the ACO_BrainSeg3D algorithm is calculated as follows: 


$$
\text { DSCmoy }=1 / 30 \sum_{i=1}^{30} \text { DSCi }
$$

Then, we obtain that we made $89 \%$ of similarity compared to the consensual segmentation. This signalize that the employed outliers segmentation algorithm generated very consistent and reproducible lesion segmentations.

Other, every patient having a total lesion load (TLL) practically similar to TLL in the case of infratentoriel segmentation for consensus.

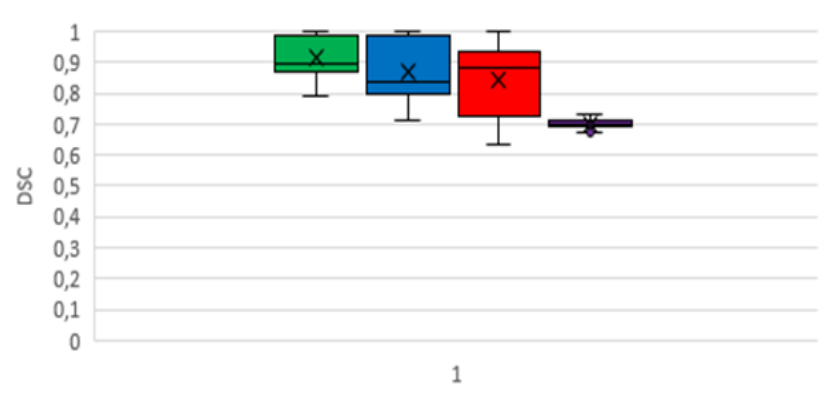

$\square$ ACO_BrainSeg3D $\square$ ACO $\square$ BrainSeg3D $\square$ consensus

Fig.10. DSC Graph for ACO_BrainSeg3D, ACO, BrainSeg3D and Consensus of inter- and intra-rater variabilities

Global, the TLL of our ACO_BrainSeg3D algorithm segmentation is significantly higher compared to the TLL of the proposed protocol used the BrainSeg3D tools compared to the segmentation of the evaluators marked in accordance with consensual segmentations where the dataset encloses a total of3316 segmented lesions with an overall TLL of $567 \mathrm{ml}$. The median TLL per patient was $15.2 \mathrm{ml}$ for each evaluator (min: $0.337 \mathrm{ml}$, max: $57.5 \mathrm{ml}$ ) [24].

The overall TLL of the proposed protocol used the BrainSeg3D tools is $389 \mathrm{ml}$. The median TLL per patient was $12.98 \mathrm{ml}$ (min: $12.1 \mathrm{ml} \max : 15.81 \mathrm{ml}$ ) with $68 \%$ of consensus.

In addition, each patient has a total lesion load (TLL) (Fig.11), depending on the consensus segmentations, the database contains approximately a total of 3280 segmented lesions with an overall TLL of $477 \mathrm{ml}$ for our proposed algorithm ACO_BrainSeg3D. Themedian TLL per patient was $15.90 \mathrm{ml}$ (min: $12.55 \mathrm{ml}$, $\max : 21.72 \mathrm{ml}$ ) with $84 \%$ of consensus (Fig.11).

According to Fig.10, we can deduce that, whereas the segmentations of each evaluator are inappropriate, because he or she extends to miss a major number of outliers, each supplementary evaluator adds noble information. And we are succeeded so that have a right result very locked to consensus based on the suggested approach ACO_BrainSeg3D, which is comprehensibly amazing to declare the diagnosis or estimate the progression of the pathology according to the current diagnostic criteria for MS.

The use of semi-automated tools compared to manual tools minimized time necessary to make the segmentation, and each processing of an image of a single patient takes approximately 2 hours, however, when we apply the semi-automatic tools combined with an optimized algorithm takes a few seconds. However, in [24] only one evaluator still needed about 300 hours (37 days if we worked 8 hours a day) to segment the tumors of the $30 \mathrm{MS}$ patients of database.

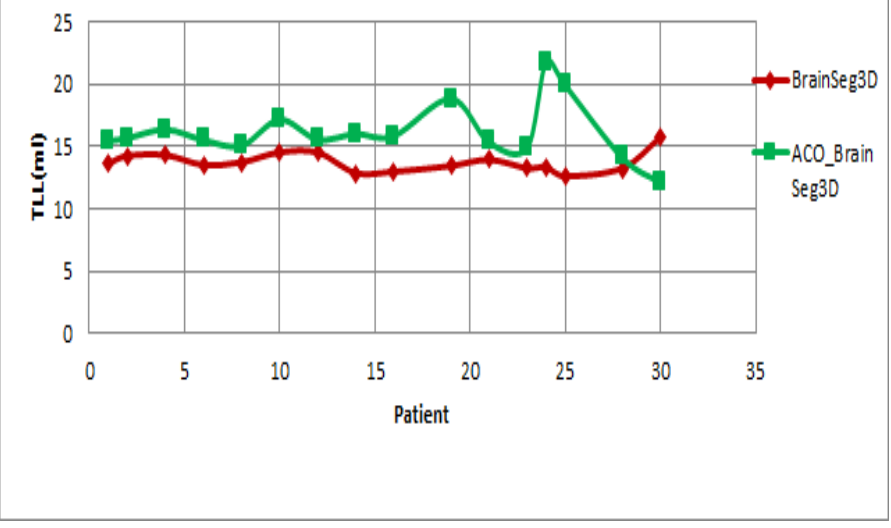

Fig.11. TLL Graph of $30 \mathrm{MS}$ patients of dataset for Protocol based on BrainSeg3D and Proposed ACO_BrainSeg3D algorithm

The execution time using the ACO_BrainSeg3D is less ( $t=$ 1 hours and 23 minutes for the all 30 patients of database) than that in the protocol based only on the BrainSeg3D software tools ( 2 hours for each patient) since we re-use images already processed by the ACO algorithm which allows us to save time in the preprocessing part and to illuminate the image and for that it becomes easier to detect MS lesions.

The ending of this propound approach is that semiautomated tools should be employed in place of manual tools based on the processed results given by the optimized ACO algorithm which facilitates visualization and detection to make lesions more visible and quick to detect. Again, to decrease the propagation of mistakes in a multi-rater consensus segmentation and so minimize intra and inter-rater variability. This approach allows us to reduce and save processing time compared to manual and semi-automatic tools from BrainSeg3D used only.

A possible failing of the current work is that the reproducibility of the segmentation of lesions used this proposed approach was evaluated with the only and the same database. It would be interesting to compare with another consensual segmentation that is done on another real database, using the same proposed approach based on the optimized ACO algorithm and the semi-automated BrainSeg3D software tools together and subsequent the tumor segmentation protocol.

Therefore, to induce and promote other searchers to replicate and spread out the performance of this work. 
It can so be marked that nearly every outliers are spotted by our propound algorithm ACO_BrainSeg3D opposite the reference, the TLL values and the DSC coefficient are probably simular. So we can tell that the BrainSeg3D software tools are efficient and capable to detect lesions of MRI images assumed by any pathology, not just multiple sclerosis, such as cancer, Alzheimer's and other pathologies. It is quick and simple to employ.

\section{CONCLUSION}

In this study, we suggested an automatic algorithm for brain outliers segmentation in MRI images. There is a segmentation problem to solve this segmentation problem; an ACO algorithm combinedwith protocol of BrainSeg3D was introduced here. The proposed algorithm used the ACO algorithm based on proposed protocol by using the tools of Brain Seg3D was derived for automatic MRI images segmentation with multiple sclerosis applied on the new databases in initial and preprocessed style as worned. We are evaluating the proposed method using public available data with synthetic brain growth image input. In both problems, our approach outperformed rivaling technics. The acquired simulations yields well results, where all the tumors have emerged in spite of there being falsely spotted outliers. This indicates that the employed lesion segmentation algorithm generated very consistent and reproducible lesions segmentations. Clearly, the novel public dataset with a more accurate and dependable reference of the segmentations is needful to push test, assimilate and rank impartially the automated tumor segmentation approaches. We can utilize the tools disposable in this software to detect tumors whatever the pathology.

\section{References}

[1] D.García-Lorenzo, S.Prima, D. L.Arnold, D. L.Collins, and C.Barillot "Trimmed-Likelihood Estimation for Focal Lesions and Tissue Segmentation in Multisequence MRI for Multiple Sclerosis", TRAN ON MEDICAL IMAGING, VOL. 30, NO. 8, AUGUST 2011.

[2] D. H. Miller, "Biomarkers and surrogate outcomes in neurodegenerative disease: Lessons from multiple sclerosis", NeuroRX., vol. 1, no. 2, pp. 284-294, Apr. 2004.

[3] D. H. Miller, R. I. Grossman, S. C. Reingold, and H. F. McFarland,"The role of magnetic resonance techniques in understanding and managing multiple sclerosis", Brain, vol. 121, no. 1, pp. 3-24, Jan. 1998.

[4] F. Nelson, A. Poonawalla, P. Hou, F. Huang, J. Wolinsky, and P.Narayana, "Improved identification of intracortical lesions in multiple sclerosis with phase-sensitive inversion recovery in combination with fast double inversion recovery MR imaging", AJNR Am. J.Neuroradiol.,vol. 28, no. 9, pp. 1645-1649, 2007.

[5] R. Zivadinov and R. Bakshi, "Role of MRI in multiple sclerosis I: Inflammation and lesions", Frontiers Biosci., vol. 9, pp. 665-683, Jan.2004.

[6] L.Devers, L.Fauvel, « TER MIASHS 2017 », Université Bordeaux.

[7] Dalenda Bouzidi, Fahmi Ghozzi, Khaled Taouil Ahmed Fakhfakh, "BrainSeg3D to detect Multiple sclerosis lesions using magnetic resonance imaging", The IEEE international conference on Design \& Test of integrated micro \& nano-Systems, ISBN:978-1-7281-54299/20/\$31.00 C2020 IEEE, Tunisia, Juin 2020.

[8] Grimaud, J., Lai, M., Thorpe, J., Adeleine, P., Wang, L., Barker,G.J., Plummer, D.L., Tofts, P.S., McDonald, W.I., Miller,D.H.(1996). Quantification of MRI lesion load in multiple sclerosis:A comparison of three computerassisted techniques. Magnetic Resonance Imaging, 14(5), 495-505, https://doi.org/10.1016/0730-72 5X(96)000185

[9] Zijdenbos, A.P., Forghani, R., Evans, A.C. (2002). Automatic"pipeline" analysis of 3-D MRI data for clinical trials: application to multiple sclerosis. IEEE Transactions on Medical Imaging, 21(10), 1280-1291.

[10] Styner, M., Lee, J., Chin, B., Chin, M., Commowick, O., Tran, H.,Markovic-Plese, S., Jewells, V., Warfield, S. (2008). 3D segmentation in the clinic: A grand challenge II: MS lesion segmentation, MIDAS Journal, 2008, 1-6.

[11]Filippi, M., Horsfield, M.A., Bressi, S., Martinelli, V., Baratti,C.,Reganati, P., Campi, A., Miller, D.H., Comi, G.(1995).Intra- andinter-observer agreement of brain MRI lesion volume measurements in multiple sclerosis. A comparison of techniques, Brain:AJournal of Neurology, 118( Pt 6), 1593-1600.

[12] Garcia-Lorenzo, D., Francis, S., Narayanan, S., Arnold, D.L., Collins,D.L. (2013). Review of automatic segmentation Methods ofmultiple sclerosis white matter lesions on conventional magnetic resonance imaging, Medical Image Analysis, 17(1),1-18, https://doi.org/10.1016/j.media.2012.09.004

[13] Llado, X., Oliver, A., Cabezas, M., Freixenet, J., Vilanova, J.C.,Quiles, A., Valls, L., Ramio-TorrentA, L., Rovira, A. (2012). Segmentation of multiple sclerosis lesions in brain MRI: A reviewof automated approaches. Information Sciences, 186(1), 164-185. https://doi.org/10.1016/j.ins.2011.10.011

[14] Vrenken, H., Jenkinson, M., Horsfield, M.A., Battaglini, M., Schijndel, R.A.V., Rostrup, E., Geurts, J.J.G., Fisher, E., Zijdenbos, A., Ashburner, J., Miller, D.H., Filippi, M., Fazekas, F.,Rovaris,M., Rovira, A., Barkhof, F., Stefano, N.D., Group,M.S. (2013). Recommendations to improve imaging and analysis of brain lesion load and atrophy in longitudinal studies ofmultiple sclerosis. Journal of Neurology, 260(10),
471,https://doi.org/10.1007/s00415-012-6762-5

[15]Dalenda Bouzidi,Fahmi Ghozzi, Ahmed Fakhfakh, “Ant colony optimisation ACO to detect Multiple sclerosis lesions using magnetic resonance imaging", Proceedings of the 4th International Conference on Recent Advances in Electrical Systems, Editors: Tarek BOUKTIR \& Rafik NEJI, ISBN: 978-9938- 9937-2-1; pages: 73-77, Hammamet, Tunisia, December 2019.

[16]J. Selvakumar, A. Lakshmi, T. Arivoli,"Brain tumor segmentation and its area calculation in brain MRimages using K-means clustering and Fuzzy C-menas algorithm", International conference on Advances inEngineering, Science, and Management(ICAESM), 2012.

[17] J. Dunn. «A fuzzy relative of the ISODATA process and its use in detecting compact well separated clusters», In J. Cybernetics, V(3:3), 32-57,1974.

[18] U.Maulik. et S.Bandyopadhay. « Genetic algorithm-based clustering technique. Pattern Recognition». V(33), 14551465., 2000.

[19]C. Senthil Singh, Rohini Paul Joseph, M.Manikandan, "Brain tumor MRI image segmentation and detection in image processing",International Journal of Research inEngineering and Technology Volume: 03 Special Issue: 01 | NC-WiCOMET-2014 | Mar 2014.

[20] A. Carass, S. Roy, A. Jog, J.L. Cuzzocreo, E. Magrath, A. Gherman, J. Button, J.Nguyen, F. Prados, C.H. Sudre, 
M.J. Cardoso, N. Cawley, O. Ciccarelli, C.A.M.WheelerKingshott, S. Ourselin, L. Catanese, H. Deshpande, P. Maurel, O.Commowick, C. Barillot, X. TomasFernandez, S.K. Warfield, S. Vaidya, A.Chunduru, R. Muthuganapathy, G. Krishnamurthi, A. Jesson, T. Arbel, O. Maier,H. Handels, L.O. Iheme, D. Unay, S. Jain, D.M. Sima, D. Smeets, M. Ghafoorian,B. Platel, A. Birenbaum, H. Greenspan, P.-L. Bazin, P.A. Calabresi, C.M.Crainiceanu, L.M. Ellingsen, D.S. Reich, J.L. Prince, D.L. Pham, "Longitudinalmultiple sclerosis lesion segmentation: resource and challenge", NeuroImage 148 (C) (2017)77-

102,http://dx.doi.org/10.1016/j.neuroimage.2016.12.064

[21] Souplet, J. C., Lebrun,C., Ayache, N., Malandain, G. "An automatic segmentation of T2-FLAIR multiple sclerosis lesions," in Proceedings of the Multiple Sclerosis Lesion Segmentation ChallengeWorkshop (MICCAI '08), New York, NY, USA, September 2008, Proceedings in The MIDAS Journal;613.

[22] Schmidt, P., Gaser, C., Arsic, M., Buck, D., Förschler, A., Berthele, A., Hoshi, M., Ilg, R.,Schmid, V. J., Zimmer, C., Hemmer, B., Mühlau, M., "An automated tool for detection of FLAIRhyperintense white matter lesions in Multiple Sclerosis", NeuroImage.2012;59(4):3774-3783.

[23] O.Ghribi, «Méthode de Détection et de Suivi de la Sclérose enPlaques via des Séquences IRM 3D », Thése de doctorat, Université de Sfax,2017.

[24]Ž. Lesjak, A.Galimzianova, A.Koren, M.Lukin, F.Pernuš, B. Likar ,Ž.Spiclin “A Novel Public MR Image Dataset of Multiple Sclerosis Patients With Lesion Segmentations Based on Multi-rater Consensus ",Springer Science+Business Media, LLC 2017.

[25] Cocosco, C.A., Kollokian, V., Kwan, R.K.S., Pike, G.B., Evans, A.C.(1997). BrainWeb: online interface to a 3d MRI simulated braindatabase. NeuroImage, 5, 425.

[26] Pham, D. (2015). Longitudinal MS lesion segmentation challenge, Last accessed: 20 oct, 2016, http://iacl.ece.jhu.edu/index.php/MSChallenge

[27] Barillot, C., Commowick, O., Guttmann, C., Styner, M., Warfield, S.(2016). MS Segmentation challenge. Last accessed: 20 oct, 2016. https://portal.fli-iam.irisa.fr/msseg-challenge/overview

[28] Akhondi-Asl, A., Hoyte, L., Lockhart, M.E., Warfield, S.K. (2014). Alogarithmic opinion pool based staple algorithm for the fusion of segmentations with associated reliability weights. IEEE Transactions on Medical Imaging, 33(10), 1997-2009.

[29] Seg3D: Volumetric image segmentation and visualization. Scientific computing and imaging institute (SCI), downloadfrom: http://www.seg3d.org

[30] Laboratory of imaging technologies http://it.fe.unili.si/tool.

[31] Nakib, A., Minery, A., Oulhadj, H. and Siarry, P., "Modified intraclass variance minimization for segmentation of retinal angiographic images". Conférence de la Société Française de Génie Biologique et Médical (SFGBM). pp. 134-135, Février 2005, Nancy.

[32] Iglesias, J., Liu, C.Y., Thompson, P., Tu, Z. (2011). RobustBrainextraction across datasets and comparison with publicly available methods, IEEE Transactions on Medical Imaging, 30(9) 16171634,https://doi.org/10.1109/TMI.2011.2138152

[33] Tustison, N., Avants, B., Cook, P., Zheng, Y., Egan, A., Yushkevich,P., Gee, J. (2010). N4ITK: Improved N3 bias correction, IEEETransactions on Medical Imaging, 29(6), 1310-1320.

[34] P.Perona, J. Malik, "Scale-space and edge detection using anisotropic diffusion”, IEEE PAMI, vol. 12, pp. 629-639, 1990.
[35]Dice, L.R., Measures of the Amount of Ecologic Association between Species, Ecology.1945;26:297-302.

[36]Zijdenbos AP, Forghani R, Evans AC. Automatic pipeline analysis of 3-D MRI data forclinical trials: application to multiple sclerosis, IEEE Trans Med Imaging 2002;21:1280-91

\section{Creative Commons Attribution License 4.0 (Attribution 4.0 International, CC BY 4.0)}

This article is published under the terms of the Creative Commons Attribution License 4.0 https://creativecommons.org/licenses/by/4.0/deed.en_US 